\title{
Sterbehilfe - welche Rolle übernimmt der Arzt?
}

\section{Hans Stalder}

Prof. Dr. med., Mitglied der Redaktion

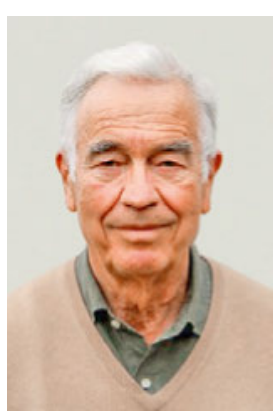

Literatur

1 StGB Art. 114 und 115

"Umgang mit Sterben und Tod». SAMW. https://www.assm.ch/ de/Ethik/Sterben-und Tod/Richtlinien-Sterben-Tod.html

Kind C. Revidierte Richtlinien zum Umrehtlinien zum Um gang mit Sterben und Tod. Schweiz Ärztezeitung. 2017;98(49) $1642-3$.

Kolatte E, Bonvallat F, Deslarzes B, et al. Euthanasie: réflexion et essai de clarification. Cahier d'Uni3, $\mathrm{Nr} 12$ Genf 2013. https:// www.unige.ch/uni3/ qui-sommes-nous/ commission/publica(a) tions
Beispiel 1: Ich habe einen Freund, der sich das Leben nehmen will und mich bittet, ihm dabei zu helfen. «Kannst du mir deine Ordonnanzpistole geben?», fragt er mich und fügt hinzu, dass mir daraus keinerlei Schwierigkeiten erwachsen würden, da Suizidhilfe laut Gesetz nur dann strafrechtlich verfolgt wird, wenn sie aus selbstsüchtigen Beweggründen erfolgt [1]. Als Arzt hätte ich ein deutlich weniger gewaltsames Mittel, wage aber nicht, ihn darauf hinzuweisen.

Beispiel 2: Mein Patient ist 80 Jahre alt und lebensmüde. Seine Frau ist bereits verstorben, seine Kinder sind nicht vor Ort, und er will nicht in ein Alters- und Pflegeheim gehen. Ich antworte, ich müsste mich nach den neuesten medizin-ethischen Richtlinien [2] vergewissern, dass sein Leiden unerträglich ist, im Zweifelsfalle einen Spezialisten zuziehen, um seine Urteilsfähigkeit bestätigen zu lassen, und eine unabhängige Drittperson müsste überprüfen, ob sein Wunsch zu sterben reiflich überlegt ist. Mein Patient fragt mich: "Warum all das? Ich habe vollstes Vertrauen zu Ihnen.» Beispiel 3: Die Patientin hat ein metastasierendes Krebsleiden und intensive Schmerzen. Sie bittet mich um Sterbehilfe. Ich antworte, ich könnte die Schmerzhemmer und Psychopharmaka erhöhen, selbst wenn sie daran verscheiden würde. Sie antwortet: «Ich möchte aber lieber mit Ihrer Unterstützung bei vollem Bewusstsein und im Kreis meiner Lieben sterben.» Die beschriebenen Szenarien wurden gewählt, um näher auf drei Punkte einzugehen, die in den neuen SAMWRichtlinien «Umgang mit Sterben und Tod» [2] entweder nicht oder nur unzureichend angegangen wurden. Gewiss sind die neuen Richtlinien ein Schritt nach vorn, hatten die bisherigen die Sterbehilfe doch nur bei unmittelbar bevorstehendem Todesfall zugelassen.

Beispiel 1 zeigt, dass in Bezug auf Sterbehilfe eine Diskrepanz zwischen unseren Standesregeln und dem Gesetz besteht, da dieses nur bei selbstsüchtigen Beweggründen eine strafrechtliche Verfolgung vorsieht [1]. Die Verschreibung von Pentobarbital ist ohne Zweifel die humanste Art der Sterbehilfe, nicht nur für die Betroffenen, sondern auch für deren Familien. Das Medikament kann nur von Ärzten verschrieben werden. Suizidhilfe ist daher ungewollt zu einer «neuen ärztlichen Aufgabe» geworden und nicht ausschliesslich «der Kompetenz drit- ter sozialer Akteure» vorbehalten [3]. Die Situation ist vergleichbar mit jener, die wir damals bei der Abtreibung erlebt haben, die besser von einem Gynäkologen durchgeführt wird als mit fragwürdigen Hilfsmitteln. Natürlich gilt, wie für die Abtreibung, dass Sterbehilfe nur praktiziert werden sollte, nachdem der Sterbewunsch ausführlich und mit Empathie erörtert wurde. Im Beispiel 2 geht es um die Autonomie des Patienten. Wer soll entscheiden, wann ein Leiden unerträglich ist? Daraus eine rein medizinische Entscheidung zu machen ist patriarchales Verhalten, eine Hilfe mehr für den Arzt als den Patienten. Ausserdem besteht die Gefahr, dass die neuen Richtlinien Leiden unnötig verlängern, vor allem wenn das Ableben unmittelbar bevorsteht, denn selbst der Hausarzt, der den Patienten gut kennt, muss bei Zweifeln an dessen Entscheidungsfähigkeit einen Spezialisten zuziehen, in wiederholten Gesprächen feststellen lassen, dass der Wunsch zu sterben nachhaltig ist, und alles noch von einer Drittperson bestätigen lassen.

Beispiel 3 zeigt, wie wir etwas scheinheilig vermeiden, bestimmte Handlungen als Euthanasie zu bezeichnen. Die Extubation eines Patienten, dessen Ableben unmittelbar bevorsteht, oder die "Sedierung bis zum Exitus» [2] in hoffnungslosen Situationen - Handlungen, die wir während unserer Arbeit im Spital alle praktiziert haben - führen aktiv den Tod herbei. Was tun, wenn der Patient bei vollem Bewusstsein und umgeben von seinen Nächsten mit ärztlicher Hilfe aus dem Leben scheiden will anstatt im medikamentös verursachten Koma? Es gibt Argumente [4] für und gegen eine Änderung des Euthanasiegesetzes [1], aber wir bevorzugen, dass diese Thematik zuerst aus ethischer Sicht diskutiert wird, bevor sie im Parlament zur Sprache kommt (was sicherlich der Fall sein wird).

Der medizinische Fortschritt in der Verlängerung und im Erhalt von Leben und gesellschaftliche Veränderungen, die patriarchales medizinisches Verhalten nicht mehr zulassen, stellen den Arzt vor neue Herausforderungen. Der Erfolg von Exit und Dignitas sollte uns zum Nachdenken veranlassen, ob unsere hippokratische Standesethik noch zeitgemäss ist. Andere Länder haben dies getan. Warum nicht auch die Schweiz? Schade, dass die Ethikkommission der SAMW nicht mit etwas mehr Mut solche sensiblen Punkte anspricht. 